\title{
LEFSCHETZ PROPERTIES OF SUBVARIETIES OF SHIMURA VARIETIES
}

\author{
T. N. VENKATARAMANA
}

\begin{abstract}
We give examples of cycle classes on certain unitary Shimura varieties which are not generated by Hecke translates of classes of Shimura subvarieties. We also give examples of cohomology classes (of low degree) which do not survive on any Shimura subvariety, thereby showing that the span of Hecke translates of Shimura subvarieties can never contain the class of an ample subvariety.
\end{abstract}

\section{INTRODUCTION}

We consider smooth projective varieties $S(\Gamma)=\Gamma \backslash X$ which are quotients of hermitian symmetric domains $X$ of non-compact type by (congruence) arithmetic subgroups $\Gamma$ of $G \mathbb{Q}$ ). Here, $G$ is a semi-simple algebraic group defined and anisotropic over $\mathbb{Q}$, and $X$ is the symmetric space of the group $G(\mathbb{R})$ of real points of $G$. These are (connected components of ) Shimura varieties. These varieties have a rich structure, and are equipped with actions of many correspondences, namely the "Hecke operators". They are defined over (some very specific) number fields $E=E(\Gamma)$ and the Galois group $G_{a l}$ acts on the $l$-adic cohomology of $S(\Gamma)$.

Suppose that $H$ is a semisimple algebraic group over $\mathbb{Q}$ with a morphism $f: H \rightarrow G$ of $\mathbb{Q}$ algebraic groups with finite kernel. Fix a maximal compact subgroup $K$ of $G$. Assume that (1) the inverse image $K_{H}$ of $K$ under $f$ is a maximal compact subgroup of $H(\mathbb{R}),(2)$ the symmetric space $Y=H(\mathbb{R}) / K_{H}$ is a Hermitian symmetric domain and (3) the imbedding $Y \rightarrow X$ of symmetric spaces induced by the map $f$ is holomorphic. Now the inverse image $\Gamma \cap H=f^{-1}(\Gamma)$ is a cocompact subgroup of $H(\mathbb{R})$, and the quotient $S_{H}(\Gamma)=\Gamma \cap H \backslash Y$ is also a smooth projective variety. We thus get a morphism $S_{H}(\Gamma) \rightarrow S(\Gamma)$ of smooth projective varieties, with finite fibres. The subvariety $S_{H}(\Gamma)$ defines an element of the cohomology of $S(\Gamma)$ (say, with $\mathbb{Q}$-coefficients); denote this element by $\left[S_{H}(\Gamma)\right]$. The classes which are $\mathbb{Q}$-linear combinations

1991 Mathematics Subject Classification. Primary 14G35; Secondary 11F41. 
of Hecke translates of classes of the type $\left.S_{H}(\Gamma)\right]$ will be referred to as special classes.

If $Z$ is a subvariety of $S(\Gamma)$ we denote by $[Z]$ the cohomology class on $S(\Gamma)$ corresponding to $Z$ by $[Z]$. Linear combinations of classes of the type $[Z]$ will be referred to as cycle classes.

The following questions arise naturally:

(1) Do the special classes yield all cycle classes?

(2) Do the special subvarieties $S_{H}(\Gamma)$ satisfy a "weak Lefschetz Property"? That is, given a cohomology class $\omega$ of degree less than the complex dimension of the variety $S(\Gamma)$, is there a special subvariety $S_{H}(\Gamma)$ and a Hecke operator $T$ on $S(\Gamma)$ such that the restriction of the translate $T^{*}(\omega)$ to the subvariety $S_{H}(\Gamma)$ is non-zero?

Note that if the class $\left[S_{H}(\Gamma)\right]$ were ample, then the restriction of $\omega$ itself would have been non-zero, and thus a strong Lefschetz property would have been satisfied. Note also that if the answer to (1) is yes, then an ample class would be a special class, and by the usual (hard) Lefschetz Theorem, the answer to (2) would be yes as well.

The simplest case when question (1) can be considered is when $S(\Gamma)$ is (the compactification of) a Hilbert modular surface. Then the only subvarieties of the form $S_{H}(\Gamma)$ are (compactifications of) embedded modular curves. From the results of $[\mathrm{HLR}],[\mathrm{MR}],[\mathrm{K}]$ it is already clear in this case that there are cycle classes in $H^{2}$ of the surface which are not special classes. Thus the answer is no in this case.

On the other hand, it is proved in $[\mathrm{V}]$ that if $S(\Gamma)$ is a quotient of the unit ball in $C^{n}$ by a suitable group $\Gamma$ (in other words, the group $G=S U(h)$ is the unit group of a suitable Hermitian form $h$ over a CM field such that $G(\mathbb{R})$ is isomorphic to $S U(1, n)$-up to compact factors), then the weak Lefschetz property does hold (as was conjectured in [HL]). Thus, when $G(\mathbb{R})=S U(1, n)$ as in the foregoing, the answer to question 2 is "yes". A similar conclusion holds (see [V]) if $G(\mathbb{R})=S O(2, n)$-again, up to compact factors. However, the answer to question (1) in these cases is "no" as shown in $[\mathrm{BR}]$.

In this talk, we show that when $S U(1, n)$ is replaced by $S U(m, n)$ with $2 \leq m \leq n$, then then answer to question (2) is in general "no". Specifically, we take $G(\mathbb{R})=S U(2,3)$. We will exhibit a holomorphic 
cohomology class of degree four in $S(\Gamma)$ - for a suitable $\Gamma$ - whose restriction to every Shimura subvariety vanishes.

In section 1 we explain the main theorem, and then add some remarks at the end .

\section{ACKNOWLEDGEMENT}

This paper is a summary of a talk given at the Conference on Algebraic Number Theory held at the Harish-Chandra Research Institute. It is an announcement of the results of [V2]. The details will appear elsewhere. We thank S.Adhikari and B.Ramakrishnan for their invitation to take part in the conference, and HRI for its excellent hospitality.

\section{The Main Result}

(2.1) Notation: Let $K$ be a totally real number field of degree $d \geq 2$ over the rational number field $\mathbb{Q}$. Let $E / K$ be a totally imaginary quadratic extension and denote by $*: z \mapsto \bar{z}$ the action of the nontrivial element of the Galois group of $E / K$. Let $h: E^{5} \times E^{5} \rightarrow E$ be the $K$-bilinear form which is Hermitian with respect to the involution $*$ : if $v, w \in E^{N}$ then $h(v, w)=\overline{h(w, v)}$. We choose $h$ as follows. Denote by $\epsilon_{i}$ the standard basis of $E^{5}$ over $E$. Write $v=\sum x_{i} \epsilon_{i}$ and $w=\sum y_{i} \epsilon_{i}$. Write $5=2+3$. Suppose that $\lambda_{i}$ (with $1 \leq i \leq$ 2) are elements of $K$ all of which are negative in some Archimedean (real, in this case) completion $K_{\infty}$ of $K$, and positive in all the other Archimedean completions of $K$. Let $\mu_{j}$ (with $1 \leq j \leq 3$ ) be elements of $K$ all of which are positive in all the archimedean imbeddings of $K$. Then

$$
\left.h(v, w)=\sum_{i=1}^{i=2} \lambda_{i} x_{i} \overline{y_{i}}+\sum_{j=1}^{j=3} \mu_{j} x_{j+2} \overline{y_{j+2}}\right) .
$$

Denote by $G$ the restriction of scalars (from $K$ ) to $\mathbb{Q}$ of the $K$ algebraic group $U(h)$. Our choice of the Hermitian form ensures us that the group $G(\mathbb{R})$ of real points of $G$ is the product of $U(2,3)$ with a $d-1$ fold product of the compact group $U(5)(d=$ degree of $K$ over $\mathbb{Q})$. Let $\Gamma \subset G(\mathbb{Q})$ be a congruence subgroup. Assume that $\Gamma$ is torsion free. The projection of $\Gamma$ to the non-compact factor $U(2,3)$ gives then a discrete co-compact subgroup of $U(2,3)$. Let $X=U(2,3) /(U(2) \times U(3))$ denote the Hermitian symmetric space associated to $U(2,3)$.Then, the quotient $S=S(\Gamma)=\Gamma \backslash X$ is a smooth projective variety, which is a 
connected component of a Shimura variety. It is clear that the dimension of $S$ is $2.3=6$.

(2.2) Notation: Suppose in the notation (3.A.6) of [CV] (with $p=2$ and $q=3$ ), that the parabolic subalgebra $\mathfrak{q}=\mathfrak{q}_{r, s}$ corresponds to $r=1$ and $s=1$. To such a parabolic subalgebra is associated (according to [VZ]) an irreducible unitary representation -denoted $A_{\mathfrak{q}}$ which has non-zero $(\mathfrak{g}, K)$-cohomology. Such a representation is called cohomological. Then, it may be seen from [CV], that the representation $A_{\mathfrak{q}}$ has holomorphic cohomology in degree four $(=r(3)+s(2-1))$.

According to a theorem of Anderson and of Li (see [And] and [Li]), the representations $A_{\mathfrak{q}}(1 \leq j \leq 3)$ occur with non-zero multiplicity in $L^{2}\left(\Gamma^{\prime} \backslash U(2,2)\right)$ for some congruence subgroup $\Gamma^{\prime}$ of $\Gamma$. This implies that there are nonzero holomorphic cohomology classes of type $A_{\mathfrak{q}}$ of degree four the variety on $S\left(\Gamma^{\prime}\right)$ for some finite cover $S\left(\Gamma^{\prime}\right)$ of $S(\Gamma)$.

We can now state the main result.

Theorem 1. There exists a holomorphic cohomology class of degree four on $S(\Gamma)$ all of whose Hecke trasnslates vanish upon restriction to every Shimura subvariety of $S(\Gamma)$.

We give a sketch of the proof:

It is easy to classify the Hermitian symmetric subdomains (of dimension at least four) of the symmetric domain $X$ associated to the group $U(2,3)$. They arise from obvious imbeddings of $U(2,2)$ in $U(2,3)$. In $[\mathrm{CV}]$ a necessary and sufficient criterion is given for a holomorphic cohomology class corresponding to a cohomological representation $\pi$ to vanish on such a subdomain, purely in terms of the minimal K-type of the representation $\pi$ and its relative position with the holomorphic tangent space of the subdomain. By using this criterion, and by using the computations in section (3.A.*) of $[\mathrm{CV}]$ it may be shown that the degree four holomorphic cohomlogy class of type $A_{\mathfrak{q}}$ can never survive when restricted to any of these subdomains. This implies that the answer to question (2) of the introduction is "no": even a weak form of Lefschetz property fails for the variety $S(\Gamma)$ associated to the the group $U(2,3)$.

Remark. As remarked by Rapoport, it is possible to use the results of $[\mathrm{Rp}]$ and $[\mathrm{Cl}]$ to produce examples of cohomology classes on quotients of the unit ball in $\mathbb{C}^{n}$-n not a prime-(by unit groups of certain division algebras of the second kind over $\mathrm{CM}$ fields) which vanish on every Shimura subvariety. So there are other examples as well, of the phenomenon of Theorem 1. 


\section{REFERENCES}

[And] G.Anderson, Theta functions and holomorphic differential forms on compact quotients of bounded symmetric domains, Duke math J.50, 1983, no.4, 1137-1170.

[BR] D.Blasius and J.Rogawski, Cohomology of congruence subgroups of $\mathrm{U}(2,1)^{p}$ and Hodge cycles on some special complex hyperbolic surfaces. in Regulators in analysis,geometry and number theory , 1-15, Progr. Math 171, Birkhauser Boston, MA, 2000.

[Cl] L. Clozel, On the cohomology of Kottwitz's arithmetic varieties, Duke Math. J. 72 (1993) 757-795.

[CV] L. Clozel and T. N. Venkataramana, Restriction of the holomorphic cohomology of a Shimura variety to a smaller Shimura subvariety, Duke Math. J. 95 (1998) 51-106.

[HLR] G.Harder, R.Langlands and M.Rapoport, Algebraische Zykeln auf HilbertBlumenthal Fla*chen, J.Reine Angew. Math., 366 (1986), 53-120.

[HL] M.Harris and J-S.Li, Lefschetz Properties of subvarieties of Shimura Varieties, J. Algebraic Geometry,7(1998), no 1,77-122.

[K] Klingenberg , Tate-Vermutung fur Hilbert-Blumenthalflachen, Invent. Math 89 (1987), no 2.

[Li] Jian-Shu Li, Nonvanishing Theorems for the Cohomology of certain arithmetic quotients. J.Reine Angew. Math. 428 (1992),177-217.

[MR] V. K. Murty and D. Ramakrishnan, Period Relations and the Tate conjecture for Hilbert modular surfaces. Invent Math.89 (1987),no. 2, 319-345.

[Rp] M. Rapoport, On the bad reduction of Shimura varieties, in Automorphic forms, Shimura varieties, and L-functions: Proceedings of a Conference held at Ann Arbor, Michigan, Vol. II, eds. L. Clozel and J. S. Milne, Perspectives in Mathematics, 11, Academic Press, New York, 1990.

[V] T.N.Venkataramana, Cohomology of Compact Locally Symmetric Spaces, Compositio Math.125 (2001), no. 2, 221-253.

[V2] T.N.Venkataramana, Some Remarks on Cycle classes on Shimura Varieties, preprint.

[VZ] D.Vogan and G.Zuckerman, Unitary Representations with Cohomology, Compositio Math.53 (1984), no. 1, 51-90.

School of Mathematics, Tata Institute of Fundamental Research, Homi Bнавна RoAd, Bombay - 400 005, INDIA.

E-mail address: venky@math.tifr.res.in 